\title{
Fatigue in martensitic 100Cr6: Relationship between rolling contact fatigue microstructural transitions and repetitive push testing
}

\author{
Jee-Hyun Kang ${ }^{\mathrm{a}}$, Pedro E. J. Rivera-Díaz-del-Castillo ${ }^{\mathrm{a}, *}$ \\ ${ }^{a}$ SKF University Technology Centre, Department of Materials Science and Metallurgy, \\ University of Cambridge, 27 Charles Babbage Road, Cambridge, CB3 OFS
}

\begin{abstract}
Repetitive uniaxial fatigue testing is introduced to reproduce a similar magnitude of compressive stress to rolling contact during bearing operation, and to investigate the associated microstructural transitions. During the test, the strain per cycle responsible for fatigue damage can be measured. The observed hardness increase suggests that the developed residual stress level is similar to that formed on ball-on-rod bearing testing. The suggested methodology would be helpful in determining the strain responsible for plastic deformation in rolling contact fatigue, as well as for appraising the quality of bearing materials employed for bearing elements.
\end{abstract}

Keywords: fatigue, martensite, steel, hardening, strain measurements, hardness measurement

\footnotetext{
${ }^{*}$ Corresponding author

Email address: pejr2@cam.ac.uk (Pedro E. J. Rivera-Díaz-del-Castillo)
} 


\section{Introduction}

During bearing operation, repetitive rolling contact between the bearing elements takes place, resulting in a form of material fatigue called rolling contact fatigue $(\mathrm{RCF})$. In the course of $\mathrm{RCF}$, damage is manifested as darketching regions, white-etching bands and white-etching areas, which are the result of microstructural alterations at the subsurface. Accordingly, their formation causes regions showing different mechanical properties than the matrix, leading to inhomogeneity across the microstructure [1].

An interesting feature during rolling contact is that the stress state varies depending on position due to a Hertzian distribution of the contact pressure. Hence, the resulting material response cannot be measured during bearing tests, although the corresponding stress state can be estimated by combining Hertzian elastic theory and cyclic plasticity [2]. In other words, conventional bearing testing methods cannot determine the experienced strain for the stress cycles due to the spatial and temporal variation in the stress state. As a result, structural fatigue tests have been carried out to obtain the strain range in cyclic torsion [3] and push-pull tests [4]. However, not enough data were obtained for stress-controlled tests, and the stress-strain responses used for calculations vary. For example, Warhadpande et al. simulated subsurface spalling during bearing operation [5] and Bhargava et al. calculated material deformation during rolling contact to predict the white-etching band morphology [6]. Although they both incorporated elastic linear kinematic plasticity, the material parametres were significantly different, especially for the yield limit (2.30 GPa versus $1.05 \mathrm{GPa}$ ) and the strain hardening slope (10 GPa versus $188 \mathrm{GPa}$ ) as shown in Fig. 1. According to tensile testing 
of $100 \mathrm{Cr} 6$ martensitic steels, the yield limit and strain hardening slope are within such ranges [7].

In this context, there is a need to obtain deformation data from stress controlled tests. In this study, a laboratory fatigue testing method is introduced to measure the plastic strain experienced by a martensitic $100 \mathrm{Cr} 6$ bearing steel. During the test, a repetitive compressive stress is applied to simulate the cyclic stress during bearing operation. The results would provide a database for the material parametres to be used in other calculations. Furthermore, they may be used as input for modelling the microstructural alteration during $\mathrm{RCF}$ via the Orowan equation, which relates the measured strain for dislocation motion during the deformation. Moreover, hardness was measured after testing and compared with the results from bearing tests. The degree of residual stress development showed a remarkable agreement with that of ball-on-rod testing. The suggested method can thus be used for the initial screening of bearing materials, which would save energy, time and cost compared with existing bearing testing methods.

\section{Theory}

Based on the Hertzian elastic contact theory, the stress state of a body in two dimensional line contact (Fig. 2) can be estimated assuming linear elasticity and no traction at the surface. There would be two normal stress components $\left(\sigma_{x}, \sigma_{z}\right)$ in the system, and a shear stress $\left(\tau_{x z}\right)$, from which the principal shear stress $\left(\tau_{1}\right)$ can be obtained [2]. The stress profiles at $x=0$ with respect to $z$ are shown in Fig. 3a. Here, $\tau_{x z}$ is zero and $\tau_{1}$ does not show its maximum at the surface; its peak lies at $(x, z)=(0,0.79 a)$ showing a value 
of $0.30 p_{0}$. In the case of $\tau_{x z}$, its maximum occurs at $(x, z)=( \pm 0.87 a, 0.50 a)$ with a value of $0.25 p_{0}$. The stress profiles at $z=0.79 a, 0.50 a$ along $x$ are shown in Fig. 3b,c, respectively. $\tau_{1}$ is believed to be responsible for plastic deformation [8] and it is seen that $\tau_{1}$ increases up to $0.30 p_{0}$ and then decreases when one cycle of over-rolling takes place. This estimation is valid up to the onset of plastic deformation.

When the material starts to deform plastically, a residual stress is generated. In a two dimensional contact configuration, the residual stress can reduce $\sigma_{x}$, resulting in a reduction of $\tau_{1}[2,9]$. However, since $\tau_{x z}$ remains constant, $\tau_{1}$ can only be reduced down to $\tau_{x z}$ maximum, $0.25 p_{0}$. Therefore, $\tau_{1}$ could only increase up to $0.25 p_{0}$ in the presence of a residual stress. Considering cyclic plasticity when the material state is between elastic and plastic shakedown [10], the stress-strain curve for each cycle would form a closed loop as shown in Fig. 4. The maximum principal shear stress $\left(\tau_{1, \max }\right)$ would then be in the range of $0.25 p_{0} \leq \tau_{1, \max } \leq 0.30 p_{0}$ depending on how much residual stress has been developed. The responsible strain for the plastic deformation would be between 0 and $\overline{A B}$ as some of the deformation is reversed. In this study, it is assumed that $\tau_{1, \max }=0.25 p_{0}$ and that the strain responsible for the plastic deformation per cycle is $\Delta \gamma_{C}=\frac{1}{2} \overline{A B}$.

\section{Material and methods}

\subsection{Materials}

$100 \mathrm{Cr} 6$ steel was used in this study. The alloy composition is shown in Table 1 . The specimens were heat-treated in the form of $15 \times 15 \times 25 \mathrm{~mm}^{3}$ blocks. They were firstly austenitised at $860{ }^{\circ} \mathrm{C}$ for 30 minutes in an Ar tube 
furnace and oil-quenched to $70{ }^{\circ} \mathrm{C}$. After 10 minutes in oil, the samples were tempered at $160^{\circ} \mathrm{C}$ for 15 minutes. The block was mechanically polished with silicon carbide papers, $6 \mu \mathrm{m}$ and $1 \mu \mathrm{m}$ diamond suspension, and colloidal silica for X-ray diffraction analysis. Data were obtained with a $2 \theta$ range of $35-125^{\circ}$ with a $0.04^{\circ}$-step and 5 s-dwell time per step employing a Philips PW1820 diffractometer using $\mathrm{Cu} \mathrm{K} \alpha$ radiation at $40 \mathrm{kV}$ and $40 \mathrm{~mA}$. By Rietveld refinement with Fullprof version 0.50 software, the measured amount of austenite was $15.3 \pm 0.3$ vol\% with (goodness of fit) ${ }^{2}=3.94$. The blocks were finally cut into cylinders of $3.43 \mathrm{~mm}$-diameter and 5.00-5.21 mm-length.

\subsection{Test conditions}

The repetitive push test in this study was set up to reproduce the response in Fig. 4 with uniaxial stress. The test consisted of uniaxial compressive stress cycles as shown in Fig. 5. During the test, a specimen is loaded up to $-p_{\max }$ during the first $30 \mathrm{~s}$, and compressive stress cycles were applied for $5 \times 10^{5}$ cycles with a frequency of $15 \mathrm{~Hz}$. Finally, unloading took place for 1 s. During the stress cycling, a minimum stress of $-0.04 \mathrm{GPa}$ was imposed in order to prevent the specimen from slipping from the stage. Note that $15 \mathrm{~Hz}$ is the maximum frequency that the employed tester manages to apply the stress stably.

$p_{\max }$ was varied from 1.0 to $3.5 \mathrm{GPa}$ with a $0.5 \mathrm{GPa}$-step. The maximum resolved shear stress $\left(\tau_{r s s, \max }\right)$ for each case can be obtained from $\tau_{r s s, \max }=$ $\frac{1}{M} p_{\text {max }}$, where $M$ is the Taylor factor equal to 2.9 for randomly oriented bodycentred cubic metals [11]. Considering Fig. 4, $\tau_{r s s, \max }$ can be considered to be equivalent to the maximum principal shear stress $\left(\tau_{1, \max }\right)$. Then, $p_{\max }$ can be related to the contact pressure $\left(p_{0}\right)$ in the bearing tests. According 
to Section 2,

$$
\begin{aligned}
\tau_{r s s, \max } & =\frac{1}{M} p_{\max }=\tau_{1, \max }=0.25 p_{0}, \\
\therefore & p_{0}=\frac{1}{0.25 M} p_{\max } .
\end{aligned}
$$

For the tested $p_{\max }$, the corresponding $\tau_{r s s, \max }$ and $p_{0}$ values are listed in Table 2.

The test employed a Mayes $100 \mathrm{kN}$ mechanical tester with the setup schematically shown in Fig. 6. The cross section of the lower stage was a $76 \mathrm{~mm}$-sided square and that of the upper stage was a $30 \mathrm{~mm}$-diameter circle. The supporters were $\sim 20 \times 20 \times 13 \mathrm{~mm}^{3}$ blocks, which were used to minimise denting on the stages during the tests. They were the 100Cr6 steel heat-treated as the tested samples, except for a 30 minutes tempering time. The specimen was polished for flat top and bottom surfaces, and placed between the supporters. Strain gages (KFG-1N-120-C1-11 from Kyowa) were attached to the side of the samples with CC-33A strain gage cement (cyanoacrylate base). The gage was the smallest available, containing a $1 \times 0.65 \mathrm{~mm}^{2}$ grid on a $4.2 \times 1.4 \mathrm{~mm}^{2}$ base. The sample was loaded by moving the upper stage while the lower stage was kept stationary. Despite the high pressure, no barreling was observed after the test, possibly due to the high hardness of the sample.

\subsection{Plastic strain data acquisition}

Examples of the obtained data from the strain gage are given in Fig. 7a. These plotted data sets represent the normal equivalents of the shear hysteresis curve in Fig. 4. To determine $\Delta \gamma_{C}, 2 \Delta \epsilon_{C}$ are firstly obtained from each curve as shown in Fig. 7b; a horizontal line on $p_{\text {mean }}=\frac{p_{\text {max }}+0.04}{2}$ 
( $p_{\text {mean }}$ and $p_{\max }$ in GPa) was drawn, and the difference of the intersecting strain values was taken. These $\Delta \epsilon_{C}$ values are defined to be $\Delta \epsilon_{C, g a g e}$. Then, $2 \Delta \epsilon_{C, g a g e}$ is plotted with respect to the number of cycles $(N)$ as shown in Fig. 8 for $p_{\max }=3.0 \mathrm{GPa}$. In all cases, $\Delta \epsilon_{C \text {,gage }}$ showed a decay with respect to $N$ as shown in Fig 8 as a result of strain hardening with time. $\Delta \epsilon_{C \text {,gage }}$ approached an asymptotic value after a certain $N$, which would correspond to the strain responsible for the plastic deformation $\left(\Delta \epsilon_{C}\right)$ for a long run bearing. However, the amount of data were not enough to fit an analytical expression, because the strain gages may not be attached all the time during the testing, possibly due to the small specimen dimension and the large test pressure. Since $2 \Delta \epsilon_{C \text {,gage }}$ approached an asymptotic value approximately after $N=100, \Delta \epsilon_{C, g a g e}$ values for $N>100$ are averaged to estimate $\Delta \epsilon_{C}$ (averaging method). In addition to this, another data set was estimated from the strain obtained with the upper stage displacement (displacement method). The normal strain $(\epsilon)$ from the upper stage position can be expressed as

$$
\epsilon=\frac{x_{0}-x_{i}}{l_{0}},
$$

where $x_{0}$ and $x_{i}$ are the initial and instantaneous position of the upper stage, respectively, and $l_{0}$ is the initial length of the specimen. The strain acquired from the stage displacement was processed the same as the strain measured with strain gage (Fig. 7b), and the $\Delta \epsilon_{C}$ values are defined as $\Delta \epsilon_{C, p o s}$. An example of $2 \Delta \epsilon_{C, p o s}$ dataset is plotted in Fig. 8 for $p_{\max }=3.0 \mathrm{GPa}$. The decay of $2 \Delta \epsilon_{C, p o s}$ over $\log N$ is very clear in all cases, and fitted to the equation:

$$
2 \Delta \epsilon_{C, \text { pos }}=b_{1} b_{2}^{\log N}+b_{3},
$$

where $b_{i}$ are fitting parameters. Note that $0<b_{2}<1$ to show the decaying 
trend. By taking 0.45 as the proportionality coefficient and multiply it to Eq. $4, \Delta \epsilon_{C, \text { gage }}$ was obtained from

$$
2 \Delta \epsilon_{C, \text { gage }}=0.45 \times 2 \Delta \epsilon_{C, \text { pos }}=0.45 b_{1} b_{2}^{\log N}+0.45 b_{3},
$$

which provided reasonable fitting for all cases. Now, $\left(\Delta \epsilon_{C}\right)$ is acquired by taking $N$ to infinity in Eq. 5. Hence,

$$
\Delta \epsilon_{C}=\left(\Delta \epsilon_{C, \text { gage }}\right)_{N=\infty}=0.225 b_{3} .
$$

Finally, this is converted to its shear equivalent via

$$
\Delta \gamma_{C}=M \Delta \epsilon_{C}
$$

For comparison, $\Delta \gamma_{C}$ values from four literature sources were collected, these were obtained from calculations and experiments. Warhadpande et al. [5] employed the finite element method to estimate the cyclic shear stressstrain hysteresis loop. They assumed that the material shows elastic linear kinematic plasticity with the mechanical parameters shown in Fig. 1a. $\Delta \gamma_{C}$ was acquired from the resulting stabilised loop. Both Bhargava et al. [6] and Hahn et al. [12] employed the same material properties of normal stressstrain hysteresis loop in Fig. 1b; $\Delta \gamma_{C}$ was obtained from $\Delta \gamma_{C}=\sqrt{3} \Delta \epsilon_{C}$, according to Von Mises material assumption. Also, Hahn et al. [3] measured $\Delta \epsilon_{C}$ from cyclic torsion tests with a constant stress amplitude and converted to $\Delta \gamma_{C}$ following the same expression. Christ et al. [4] employed a symmetric push-pull fatigue test. The stress amplitude was taken to be equivalent to $\frac{1}{2} p_{\max }$ and the corresponding $p_{0}$ was calculated with Eq. 2 and $\Delta \epsilon_{C}$ was converted to $\Delta \gamma_{C}$ via Eq. 7 . 
In addition to $\Delta \gamma_{C}$, the maximum plastic strain $\left(\epsilon_{p, \max }\right)$ has been acquired for each cycle during the testing. The total maximum strain $\left(\epsilon_{\max }\right)$ is defined and obtained from the strain gage data as shown in Fig. 7b. From $\epsilon_{\text {max }}$, its plastic contribution was obtained by subtracting the elastic part;

$$
\epsilon_{p, \max }=\epsilon_{\max }-\left(\frac{p_{\text {mean }}}{E}\right),
$$

where $E$ is the elastic modulus taken as $210 \mathrm{GPa}$.

\subsection{Microscopy and microindentation}

After the tests, the specimens were sectioned into their longitudinal direction. They were mechanically ground with silicon carbide papers, polished with $6 \mu \mathrm{m}$ and $1 \mu \mathrm{m}$ diamond suspensions. After additional polishing with colloidal silica, hardness was measured with microindentation. $9 \times 4$ arrays of equally spaced indentations were made alongside the sample (Fig. 9) employing a Mitutoyo MVK-H2 tester with a 2 kg-load and 15 s-dwell time. Finally, the samples were etched with $2 \%$ nital and their microstructures were analysed with optical microscopy (OM) and scanning electron microscopy (SEM) employing a Leica DM 2500M microscope, and a JEOL 5800LV. SEM Imaging adopted a $10 \mathrm{keV}$-electron beam with 5-10 mm-working distance.

\section{Results and discussion}

\subsection{Microstructure}

Typical microstructures before and after testing are respectively shown in Figs. 10 and 11. No clear difference in the matrix is appreciated after testing, even nearby inclusions by OM and SEM, although microcracks were more 
frequently observed after testing. Under higher compressive stress $\left(p_{\max } \geq\right.$ 3.0 GPa), cracks were observed around some inclusions (Fig. 12). However, further study is required to confirm whether cracking is the result of loading or it already existed before.

\subsection{Material response $\left(\Delta \gamma_{C}\right.$ and $\left.\epsilon_{p, \max }\right)$}

The progress in normal strain per cycle with the number of cycles $(N)$ for different pressure conditions is shown in Fig. 13. The fitting parameters for the displacement method to obtain the analytical expression for $\Delta \epsilon_{C, \text { pos }}$ and $\Delta \epsilon_{C, \text { gage }}$ (Eqs. 4,5$)$ are listed in Table 3. The decaying trend of $2 \Delta \epsilon_{C}$ is well captured both by the gage and the machine crosshead. The resulting $\Delta \gamma_{C}$ values obtained by both averaging and displacement methods are shown in Fig. 14 and compared with reported values $[3-6,12]$. The measured $\Delta \gamma_{C}$ in this study was always in the range of $2 \times 10^{-4}-6 \times 10^{-4}$ for the pressure of interest except when $p_{0}=4.82 \mathrm{GPa} . \Delta \gamma_{C}$ values obtained via the averaging method were always higher than those via the displacement method. This is because the averaged strain included the values before reaching the asymptote. Especially at $p_{0}=4.82$, the strain gage was disconnected from the sample from the early stage (Fig. 13f), resulting in larger differences depending on the obtaining method. Ignoring this value, it seems that the measured $\Delta \gamma_{C}$

reaches an asymptotic value of $3.5 \times 10^{-4}$ (displacement method) or $5.5 \times 10^{-4}$ (averaging method) when $p_{0}>2.5 \mathrm{GPa}$. Although the displacement method has an advantage over the averaging method in this regard, $\Delta \gamma_{C}$ estimated from fitting the stage position data cannot incorporate complex behaviour of the machine stiffness $[13,14]$. Hence, both methods has been employed in this study; the actual strain range after a number of cycles would reach the 
value lower than $5.5 \times 10^{-4}$ predicted via the averaging method and near to $3.5 \times 10^{-4}$ obtained by the displacement method. Despite the different acquisition methods of previous studies [3-5], all data showed $\Delta \gamma_{C}$ in the order of $10^{-4}$, supporting the results obtained in this study. Only the value used by Bhargava et al. [6] and Hahn et al. [12] for their calculations is significantly higher (Fig. 14b).

$\epsilon_{p, \max }$ is plotted in Fig. 15. It is interesting to note that $\epsilon_{p, \max }$ does not increase but approaches zero at the beginning of the test in spite of the compressive nature of the stress during the test; this could be ascribed to the volume expansion due to stress-induced martensitic transformation of retained austenite. Considering the initial amount of austenite (15.3 vol\%), the maximum plastic strain due to the transformation would be 0.023 [15]. The order of the estimated strain is similar to $\epsilon_{p, \max }$ at $N=1$ in our study, which supports the high possibility of martensitic transformation during early stages of deformation. Further confirmation would be possible via X-ray diffraction analysis in the future. By attributing the positive $\epsilon_{p, \max }$ to the stress-induced transformation, the dependence of the initial $\epsilon_{p, \max }$ on $p_{\max }$ suggests that the fraction of austenite transforming to martensite would be governed by the magnitude of $p_{\max }$; less austenite transforms into martensite with lower $p_{\max } . \epsilon_{p, \max }$ decreases faster with $N$ for larger values of $p_{\max }$. For all conditions, except $p_{\max }=3.5 \mathrm{GPa}, \epsilon_{p, \max }$ overlapped at $N \approx 4 \times 10^{2}$ with a value of $\sim 0.006$. In the case of $p_{\max }=3.5 \mathrm{GPa}$, not enough data were obtained due to severe deformation. 


\subsection{Hardness}

Hardness profiles after testing are shown in Fig. 16. Hardness hardly changes for $p_{\max } \leq 1.5 \mathrm{GPa}$, but increases nonlinearly with $p_{\max }$ when $p_{\max } \geq 2.0 \mathrm{GPa}$. The hardness increase is attributed to residual stress development during the tests [16], possibly resulting from both (i) martensitic transformation of retained austenite and (ii) dislocation generation and rearrangement. The lower hardness at the surface is attributed to stress relaxation or may represent the undeformed regions due to friction between the sample and the supporter [17]. However, it is clear that these regions are only limited to $z \leq 0.1 l_{f}$ and $z \geq 0.9 l_{f}$ at high pressure $\left(p_{\max } \geq 3.0 \mathrm{GPa}\right)$, which agrees well with the fact that barreling has not occurred during testing. This implies that the friction between the sample and the supporter did not significantly affect the stress state at the central region $\left(0.1 l_{f} \leq z \leq 0.9 l_{f}\right)$.

The hardness values were averaged excluding $\frac{z}{l_{f}}=0.1,0.9$ and the resulting hardness change $(\Delta \mathrm{H} V)$ is plotted with respect to $p_{0}$ in Fig. 17, where the results from a ball-on-rod test presented in the accompanying paper [18] are also shown. The data from ball-on-rod testing corresponds to the maximum hardness at the subsurface. The correspondence in $\Delta \mathrm{HV}$ implies that the magnitude of residual stress is remarkably similar. This suggests that $\Delta \gamma_{C}$ obtained in this study can reasonably be taken as that at the subsurface, representing the maximum subsurface stress developed in bearing element testing. Moreover, it suggests the possibility for the newly proposed method to be employed for the initial screening of new bearing materials. 


\section{Conclusions}

Considering cyclic rolling contact during bearing operation, a repetitive push testing method employing a conventional uniaxial fatigue tester was proposed. Tests with 100Cr6 martensitic steel showed that

(i) Microstructural change in the matrix was hardly detected by optical microscopy, while some inclusions were observed to be accompanied by cracks with $p_{0} \geq 3.0 \mathrm{GPa}$.

(ii) The subsurface strain range per stress cycle increases according to the compressive stress and reaches $\sim 3.5 \times 10^{-4}$ with $p_{0} \geq 2.5 \mathrm{GPa}$.

(iii) The plastic strain decays from the beginning of the test, suggesting that retained austenite transforms to martensite from the first cycles, which needs futher confirmation.

(iv) The amount of transformed austenite seems to increase with the applied load.

(v) The degree of subsurface hardness increase obtained from the repetitive push test is similar to that from a ball-on-rod rolling contact fatigue test.

Overall, this study suggests the possibility that the repetitive push test could produce a stress state that triggers similar microstructural alterations to those resulting from rolling contact fatigue in bearing steels. 


\section{Acknowledgments}

This work was supported by SKF Engineering \& Research Centre and financed by SKF AB. The authors would like to express a sincere gratitude to Prof. A. L. Greer for the provision of laboratory facilities at the University of Cambridge.

\section{Reference}

[1] J.-H. Kang, B. Hosseinkhani, P. E. J. Rivera-Díaz-del Castillo, Materials Science and Technology 28 (2012) 44-49.

[2] K. L. Johnson, Contact Mechanics, Cambridge University Press, 1987.

[3] G. Hahn, V. Bhargava, Q. Chen, Metallurgical and Materials Transactions A 21 (1990) 653-665.

[4] H. J. Christ, C. Sommer, H. Mughrabi, A. P. Voskamp, J. M. Beswick, F. Hengerer, Fatigue and Fracture of Engineering Materials and Structures 15 (1992) 855-870.

[5] A. Warhadpande, F. Sadeghi, M. N. Kotzalas, G. Doll, International Journal of Fatigue 36 (2012) 80-95.

[6] V. Bhargava, G. T. Hahn, C. A. Rubin, Metallurgical Transactions A 21 (1990) 1921-1931.

[7] A. T. W. Barrow, J. H. Kang, P. E. J. Rivera-Díaz-del-Castillo, Acta Materialia 60 (2012) 2805-2815. 
[8] G. E. Dieter, D. Bacon, in: Mechanical Metallurgy, McGraw-Hill series in materials science and engineering, McGraw-Hill, 1988, p. 77.

[9] J. E. Merwin, K. L. Johnson, Proceedings of the Institution of Mechanical Engineers 177 (1963) 676-690.

[10] K. L. Johnson, Proceedings of the Institution of Mechanical Engineers 203 (1989) 151-163.

[11] U. F. Kocks, Metallurgical and Materials Transactions 1 (1970) 11211143.

[12] G. T. Hahn, V. Bhargava, C. A. Rubin, Q. Chen, K. Kim, Journal of Tribology 109 (1987) 618-626.

[13] J. E. Hockett, P. P. Gillis, International Journal of Mechanical Sciences 13 (1971) 251-264.

[14] J. H. Holbrook, J. C. Swearengen, R. W. Rohde, in: Mechanical Testing for Deformation Model Development, volume 765 of ASTM STP, Americal Society for Testing and Materials, 1982, pp. 80-101.

[15] H. K. D. H. Bhadeshia, ISIJ International 42 (2002) 1059-1060.

[16] J. Frankel, A. Abbate, W. Scholz, Experimental Mechanics 33 (1993) 164-168.

[17] G. E. Dieter, D. Bacon, in: Mechanical Metallurgy, McGraw-Hill series in materials science and engineering, McGraw-Hill, 1988, p. 520. 
[18] J.-H. Kang, R. H. Vegter, P. E. J. Rivera-Díaz-del Castillo, Rolling contact fatigue in martensitic 100Cr6: subsurface hardening and crack formation, 2014. Accepted in Materials Science and Enginnering A. 
(a)

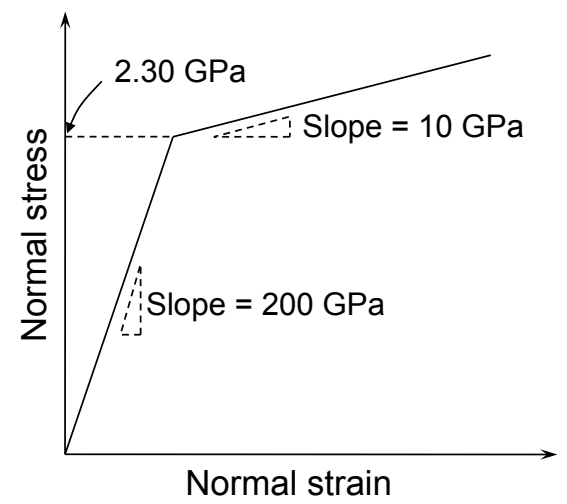

(b)

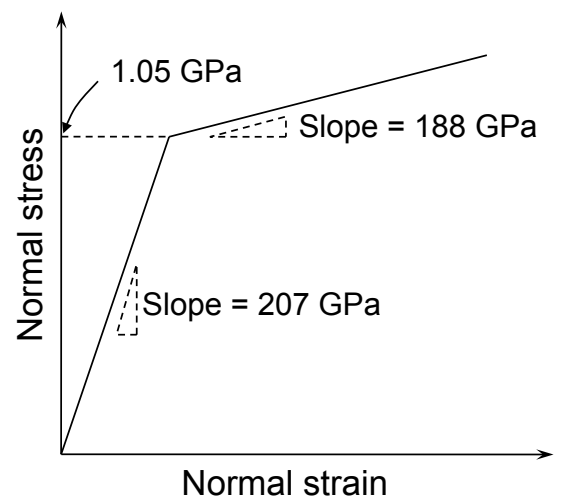

Figure 1: Material parametres used for elastic linear kinematic plasticity assumption in (a) Warhadpande et al. [5], and (b) Bhargava et al. [6].

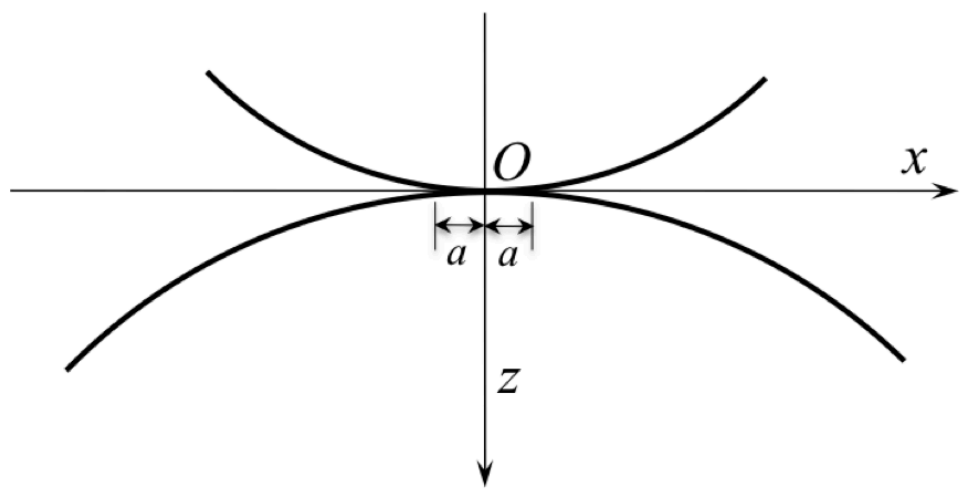

Figure 2: A two dimensional contact between two bodies resulting in a line contact with $2 a$ thickness. 
(a)

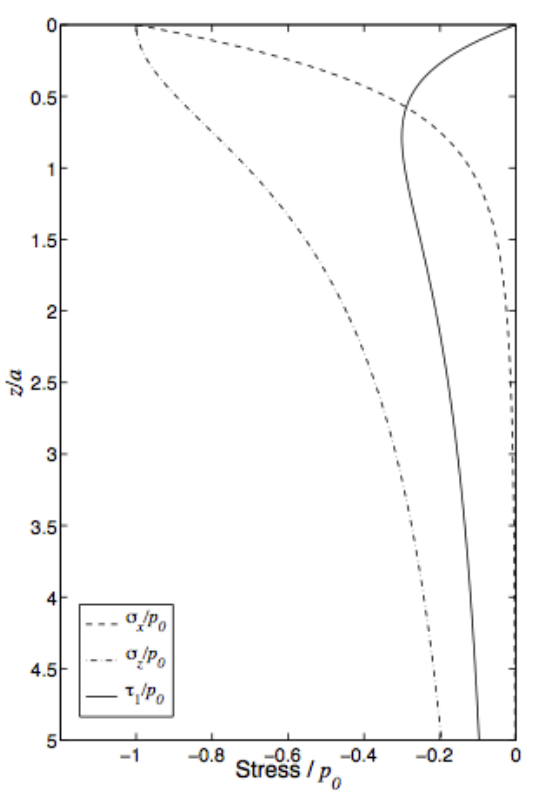

(b)

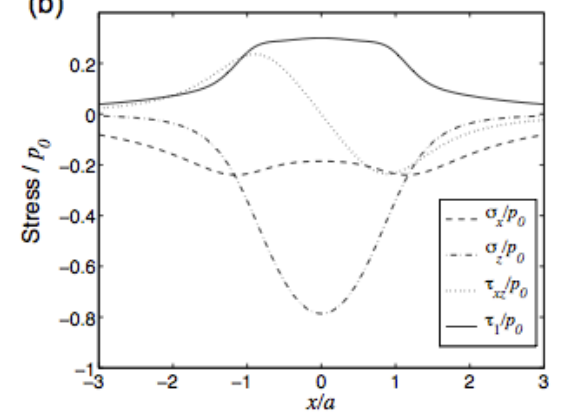

(c)

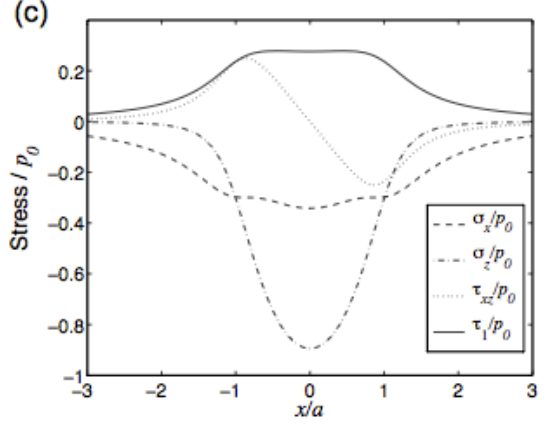

Figure 3: Stress distribution for a two dimensional line contact at (a) $x=0$, (b) $z=0.79 a$, and (c) $z=0.50 a$. 


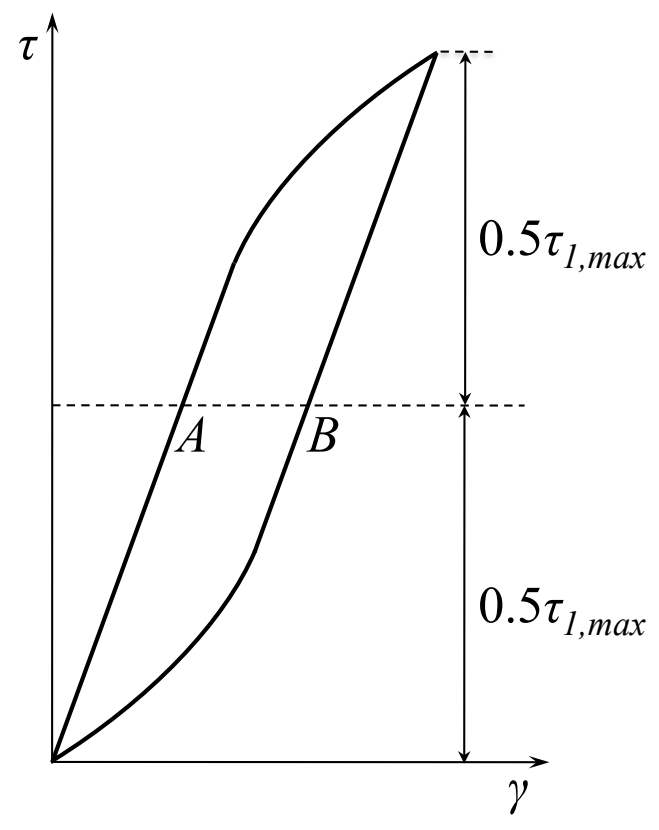

Figure 4: Hysteresis stress-strain curve for the material in the state of cyclic plasticity during bearing operation $\left(\tau\right.$ : shear stress, $\gamma$ : shear strain, $\tau_{1, \text { max }}$ : maximum principal shear stress). 


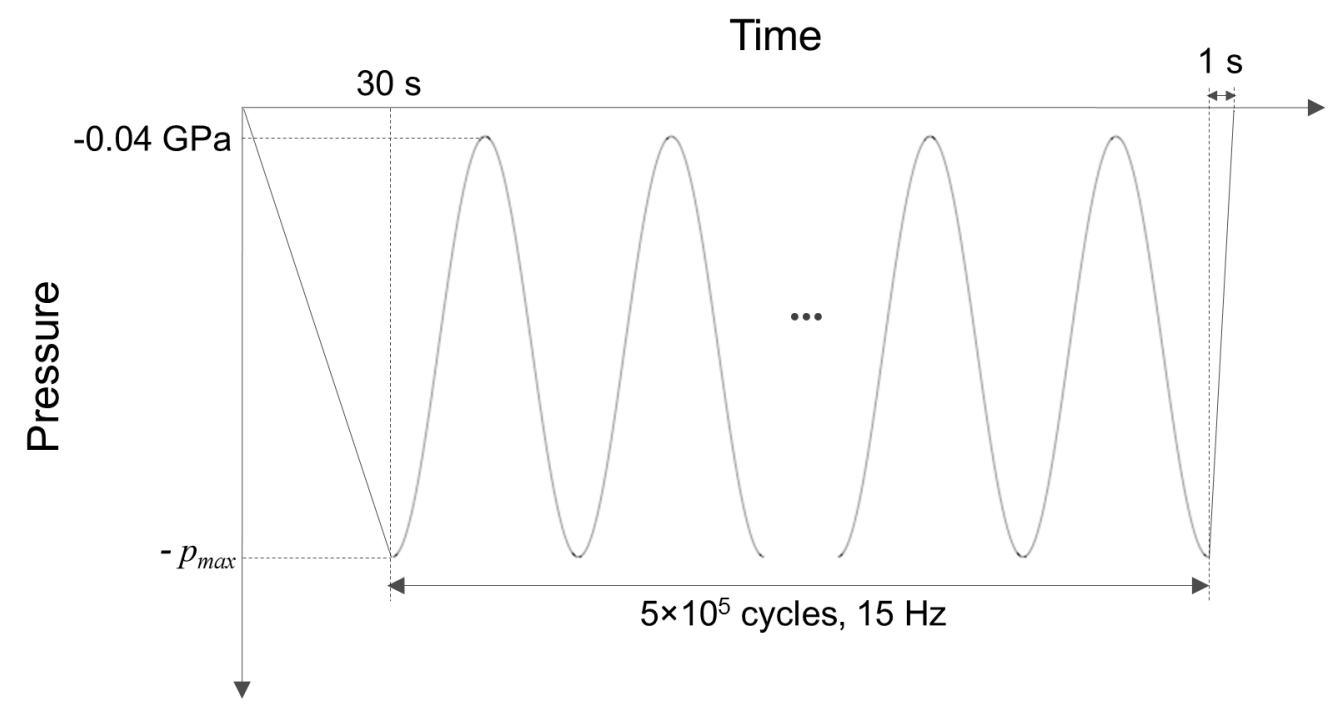

Figure 5: Schematic test cycles for the repetitive push test.

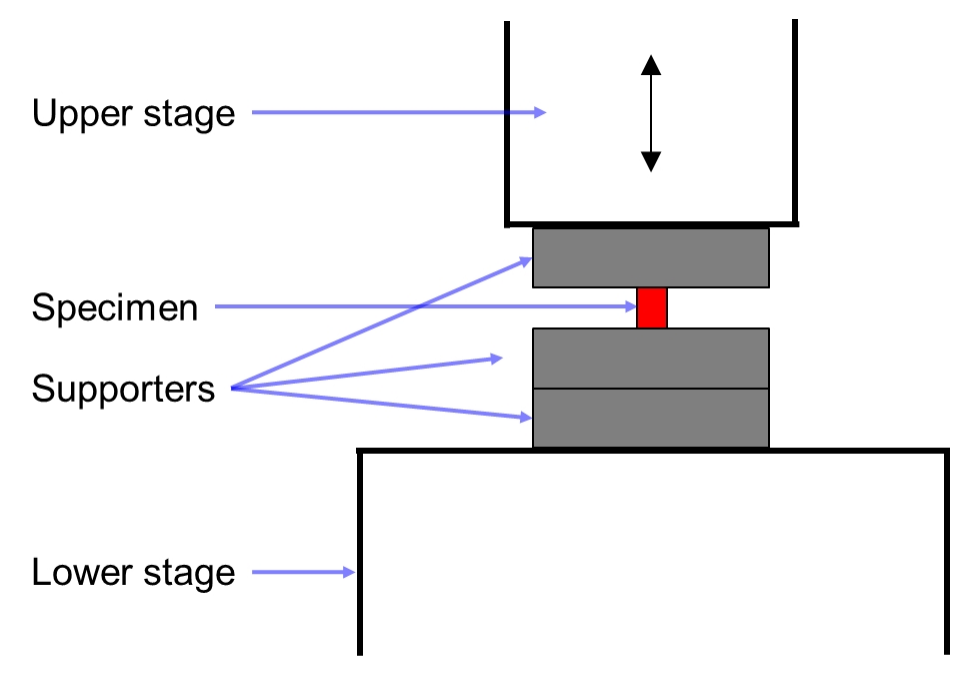

Figure 6: Schematic cross sectional view of the repetitive push test setup. 
(a)

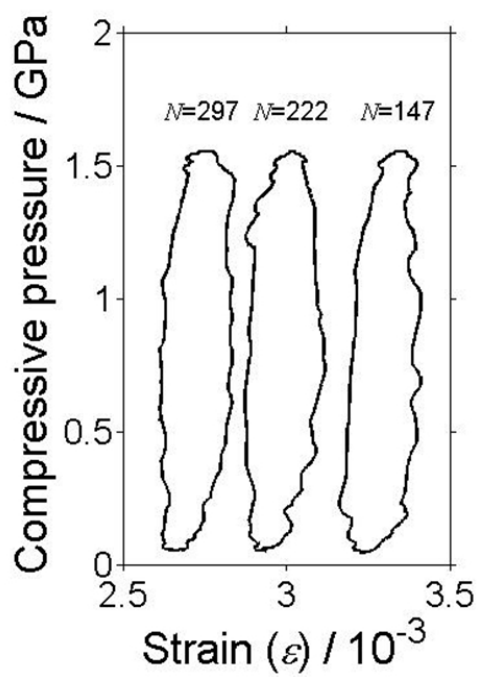

(b)

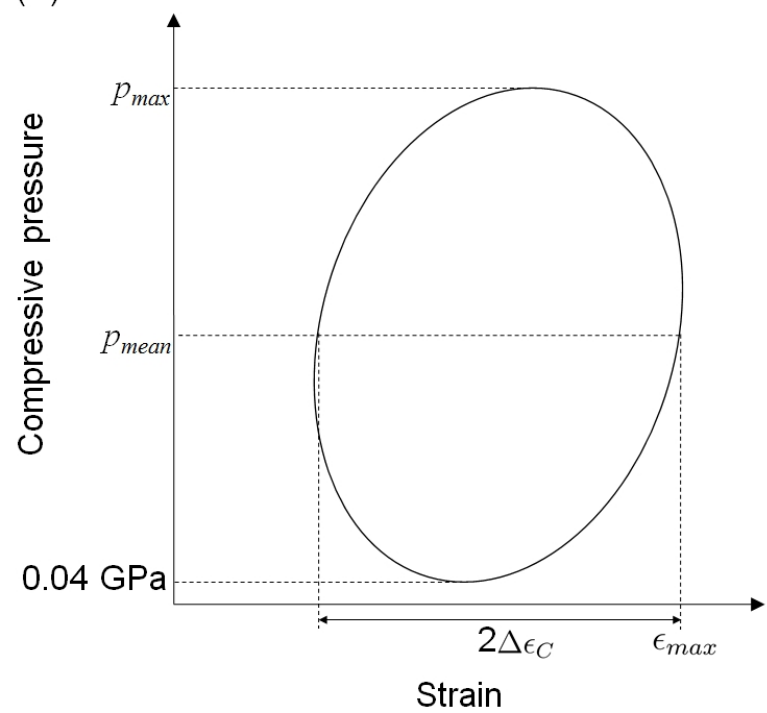

Figure 7: (a) Examples of the stress-strain data obtained from the strain gage for $p_{\max }=1.5 \mathrm{GPa}, N=147,222,297$. (b) Schematic showing how $2 \Delta \epsilon_{C}$ was obtained from pressure-strain data. 


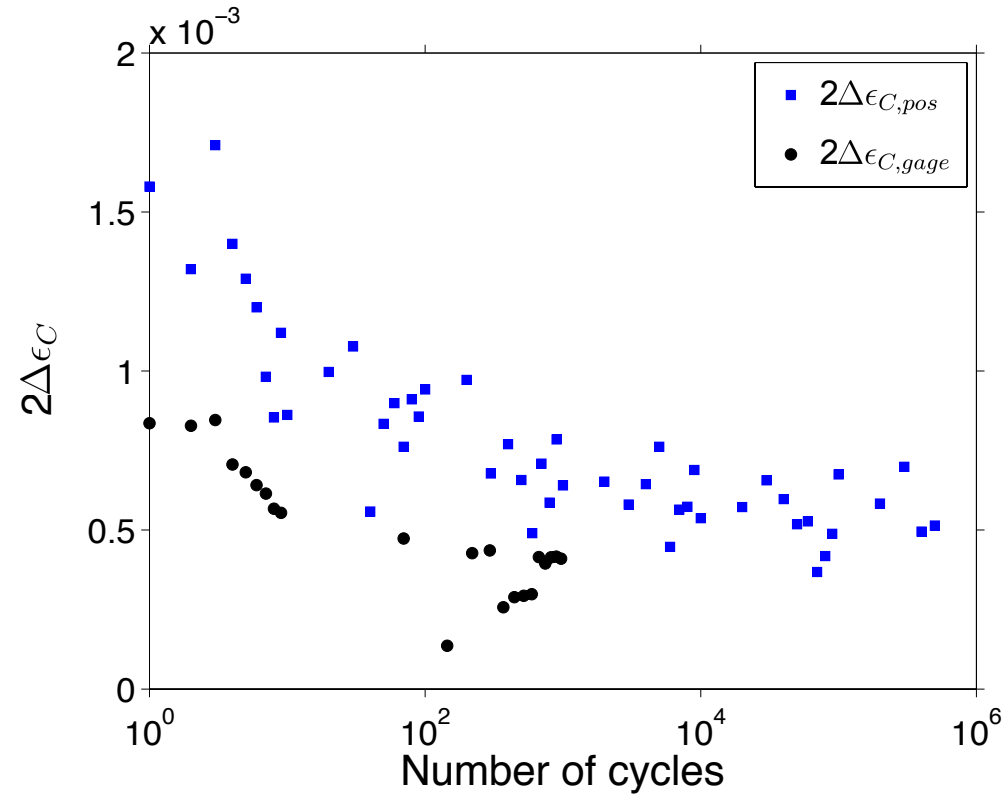

Figure 8: An example of $2 \Delta \epsilon_{C, \text { gage }}$ and $2 \Delta \epsilon_{C, \text { pos }}$ variation with respect to $N$ for $p_{\max }=3.0$ GPa.

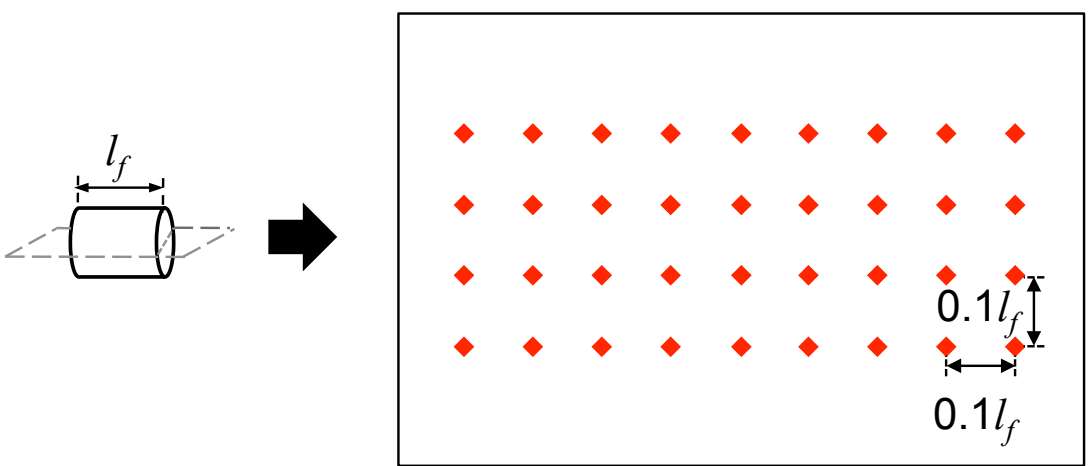

Figure 9: Schematic showing the indentation arrays made for hardness in each sample. $l_{f}$ is the final length of the sample after the test. 

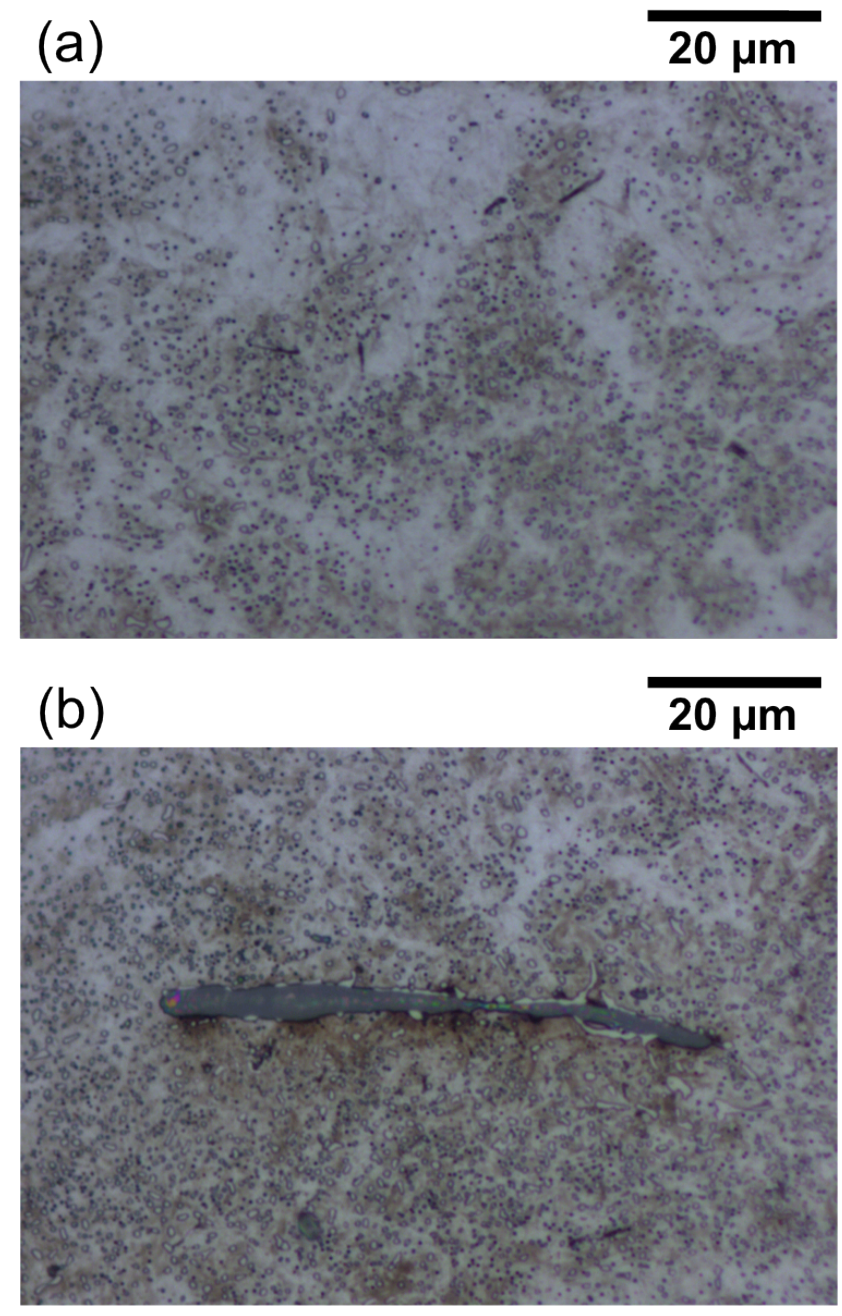

Figure 10: Optical micrographs of (a) microcracks and (b) the region adjacent to an inclusion before testing. 

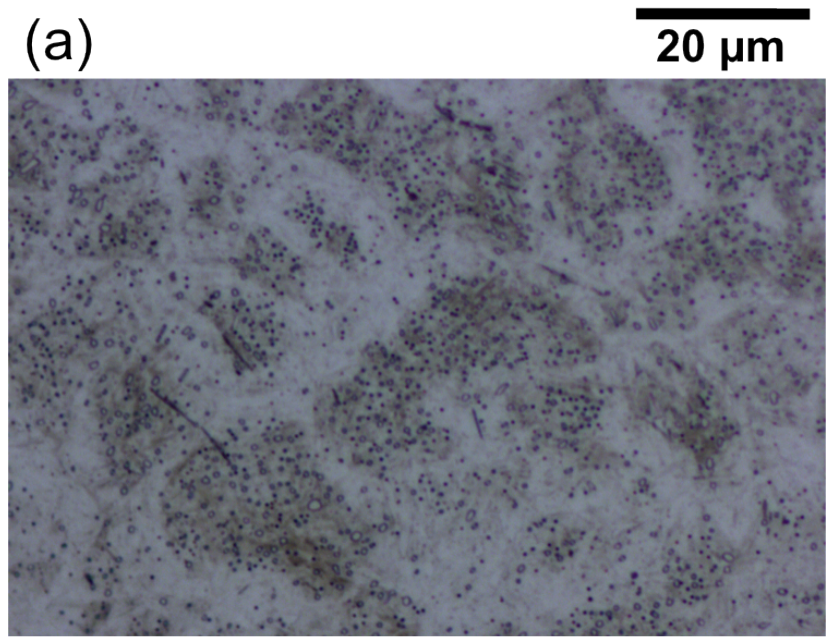

(b)

$20 \mu \mathrm{m}$

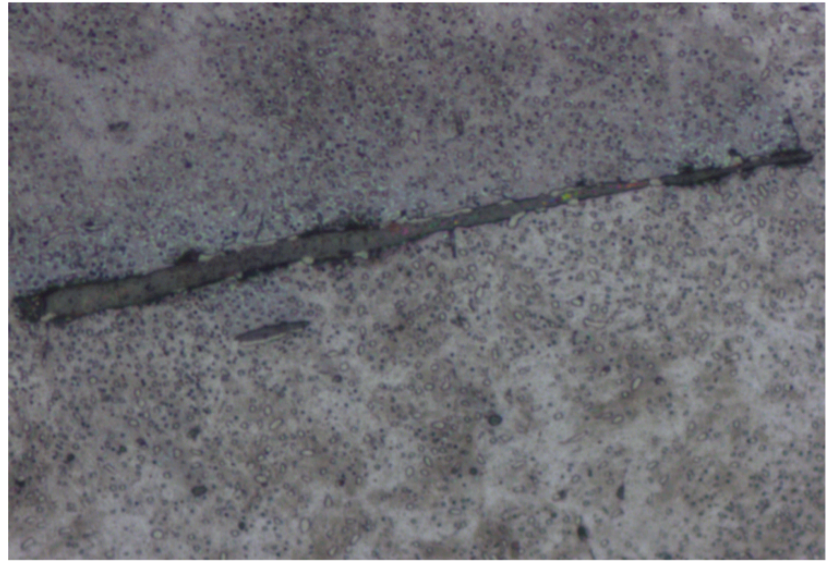

Figure 11: Optical micrographs of (a) microcracks and (b) the region adjacent to an inclusion after testing; (a) with $p_{\max }=1.0 \mathrm{GPa}$ and (b) with $p_{\max }=1.5 \mathrm{GPa}$. Stress was applied in the horizontal direction. 


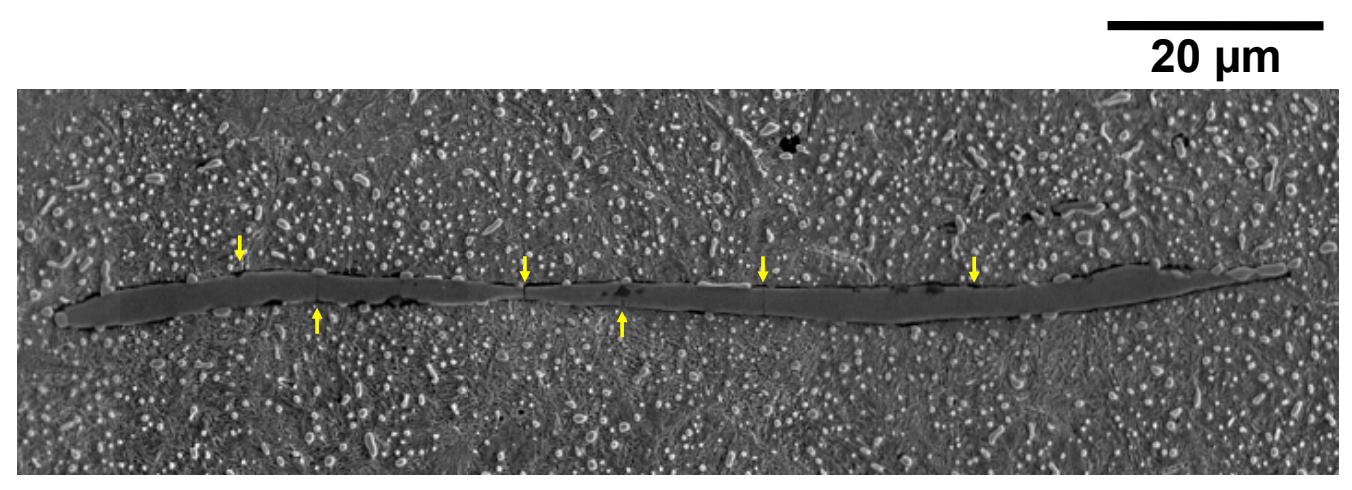

Figure 12: A secondary electron image of an inclusion containing cracks indicated with arrows. The sample was tested with $p_{\max }=3.5 \mathrm{GPa}$. 


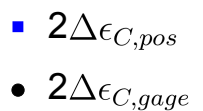

(a)

(b)
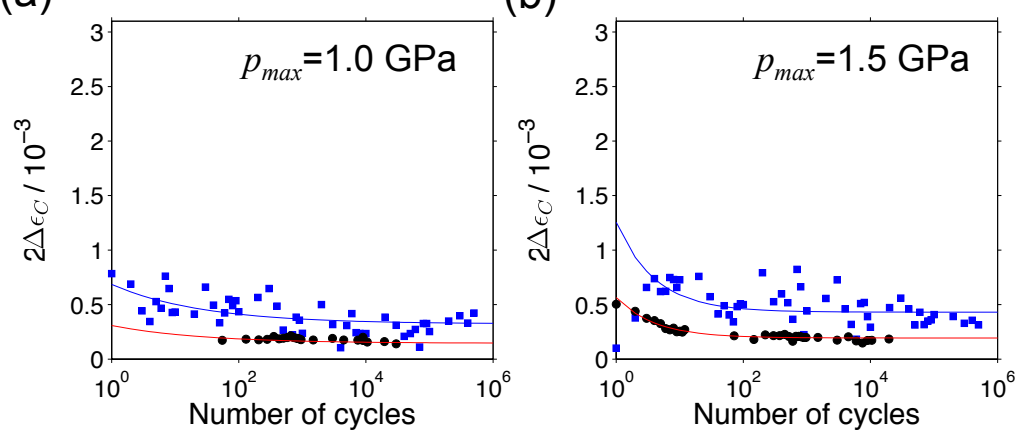

(c)

(d)
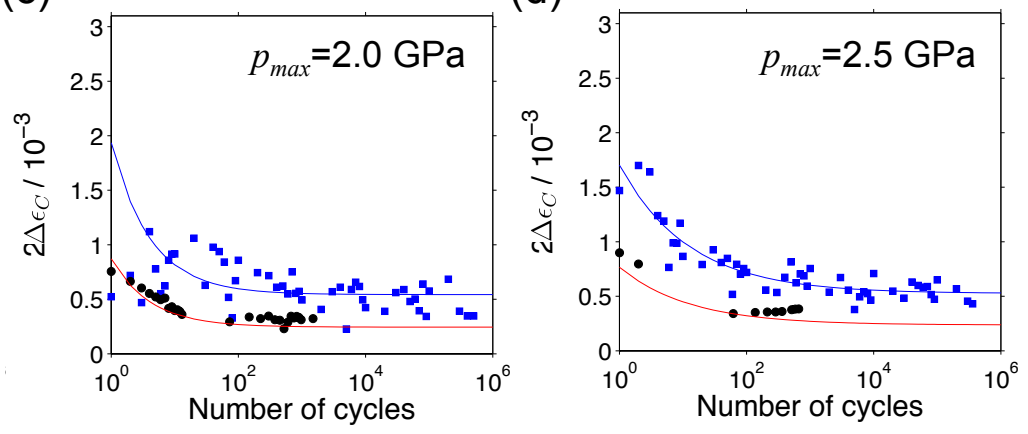

(e)

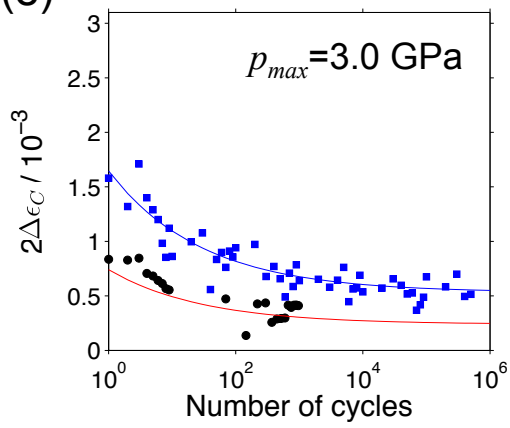

(f)

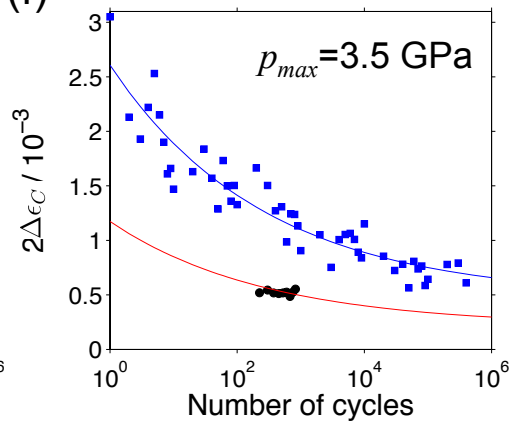

Figure 13: $2 \Delta \epsilon_{C}$ data (in markers) obtained from the lower stage position $\left(2 \Delta \epsilon_{C, p o s}\right)$ and the strain gage $\left(\Delta \epsilon_{C, g a g e}\right)$ for different $p_{\max }$. Lines show fitted curves according to Eqs. 4 and 5 . 
(a)

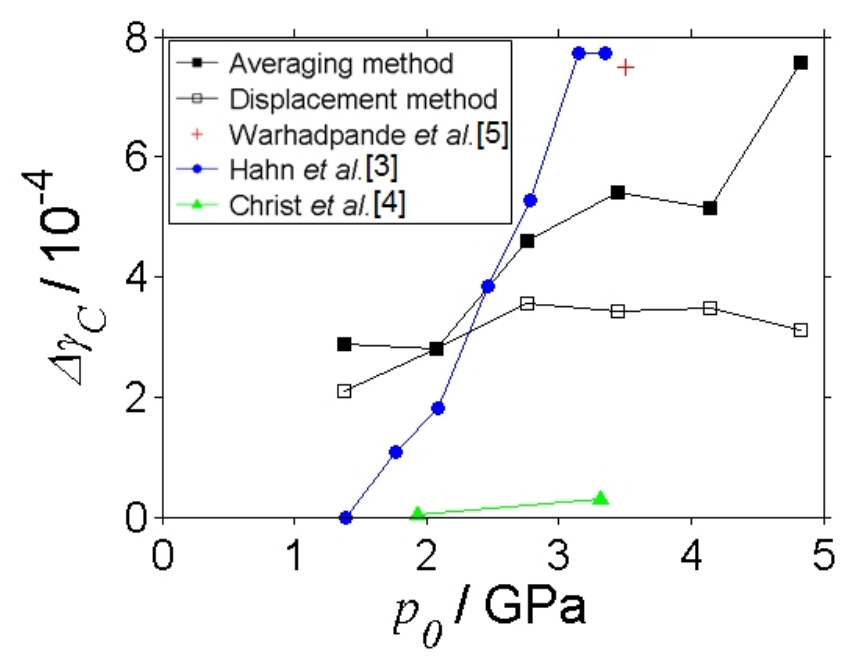

(b)

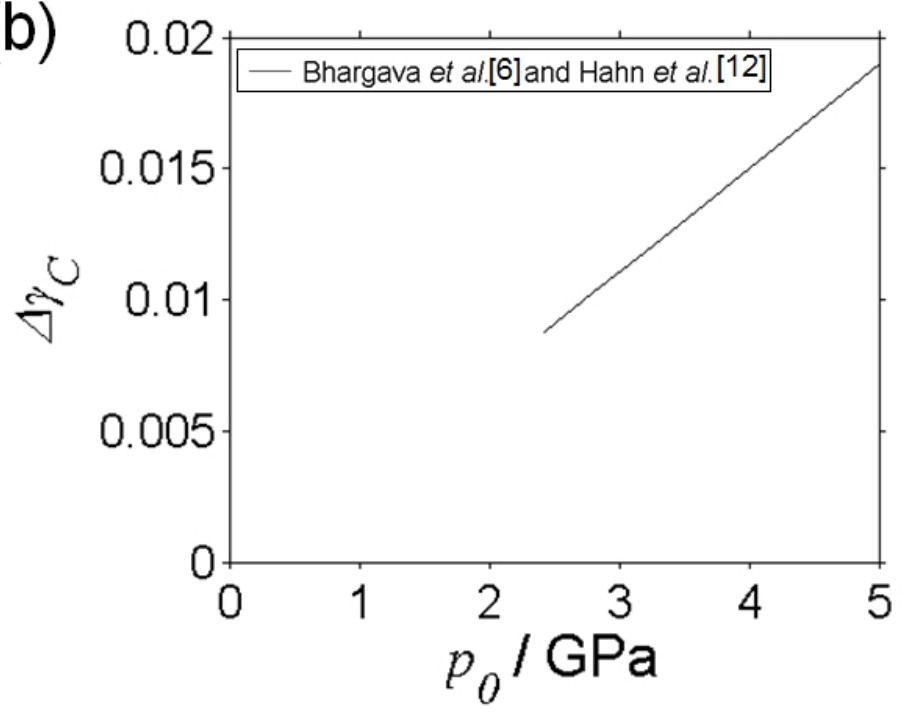

Figure 14: Measured $\Delta \gamma_{C}$ values and other reported values. Note the scale difference in (a) and (b). 


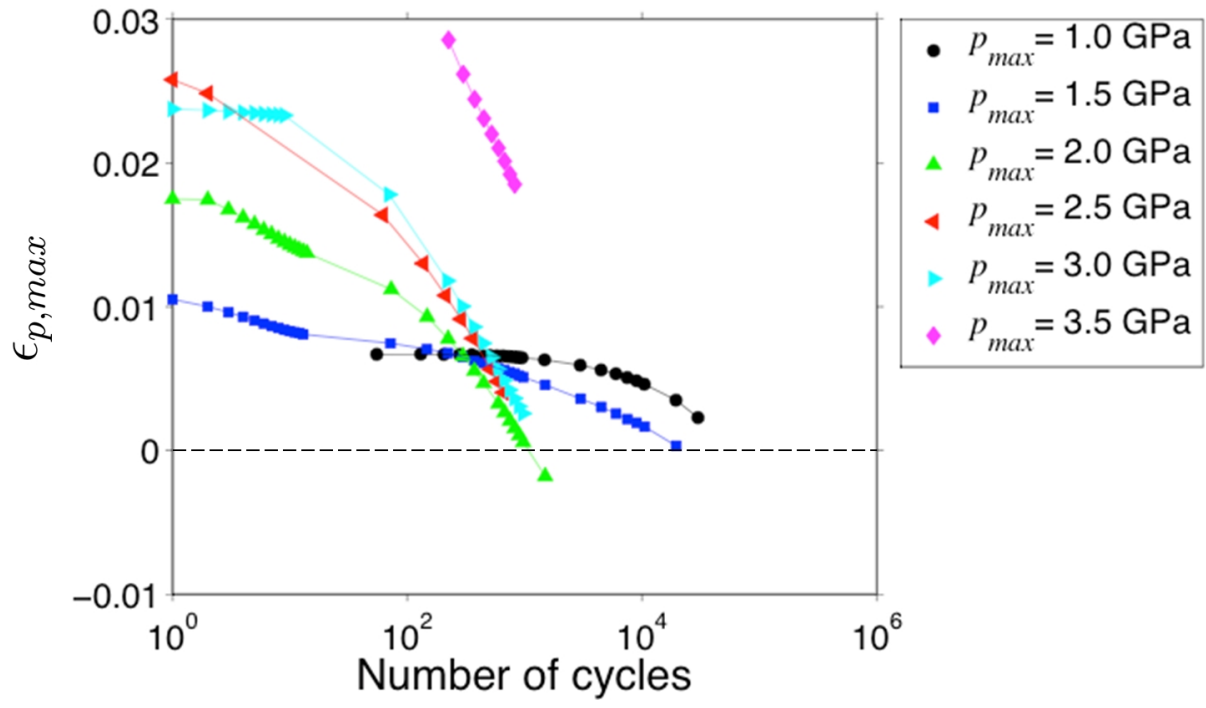

Figure 15: Maximum normal plastic strain $\left(\epsilon_{p, \max }\right)$ with respect to number of cycles for the different stress conditions. 


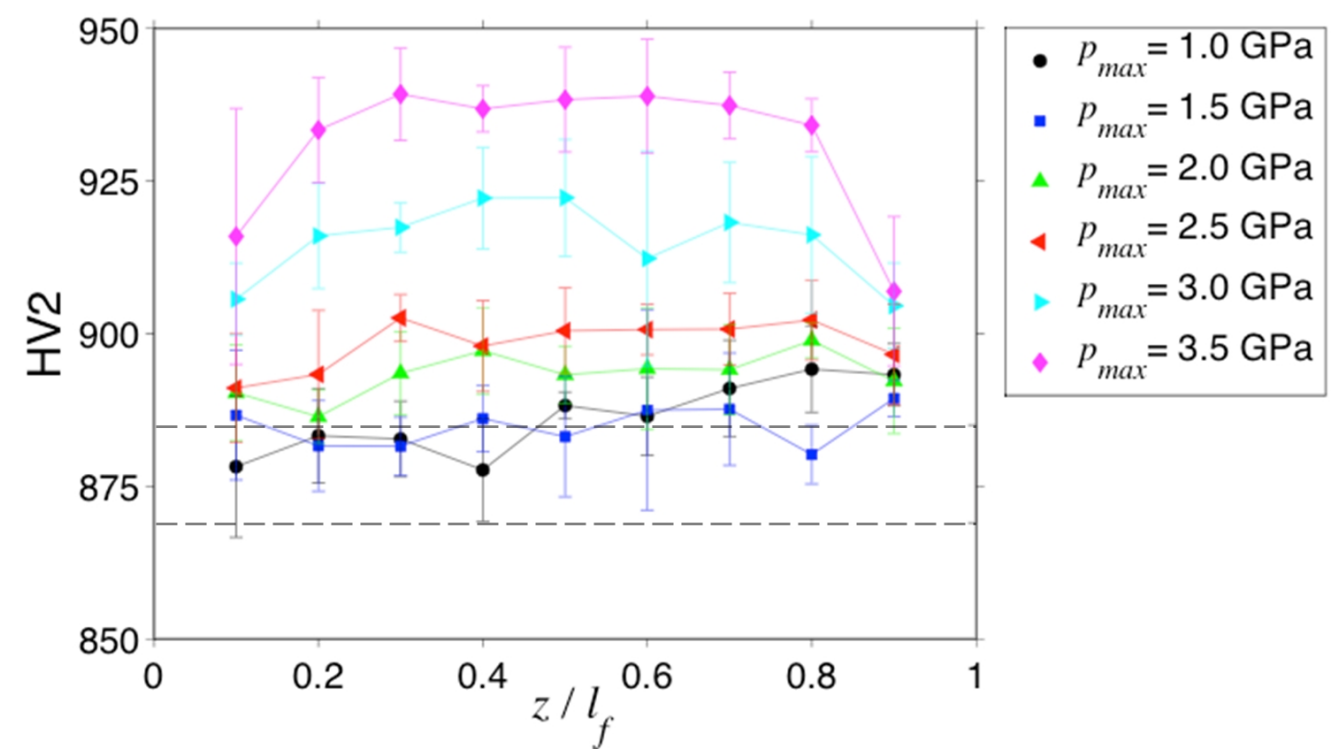

Figure 16: Harndess profile with respect to the normalised depth. $z$ is the depth from the surface and $l_{f}$ is the final specimen length. The two dotted horizontal lines show the hardness range of the untested specimen. 


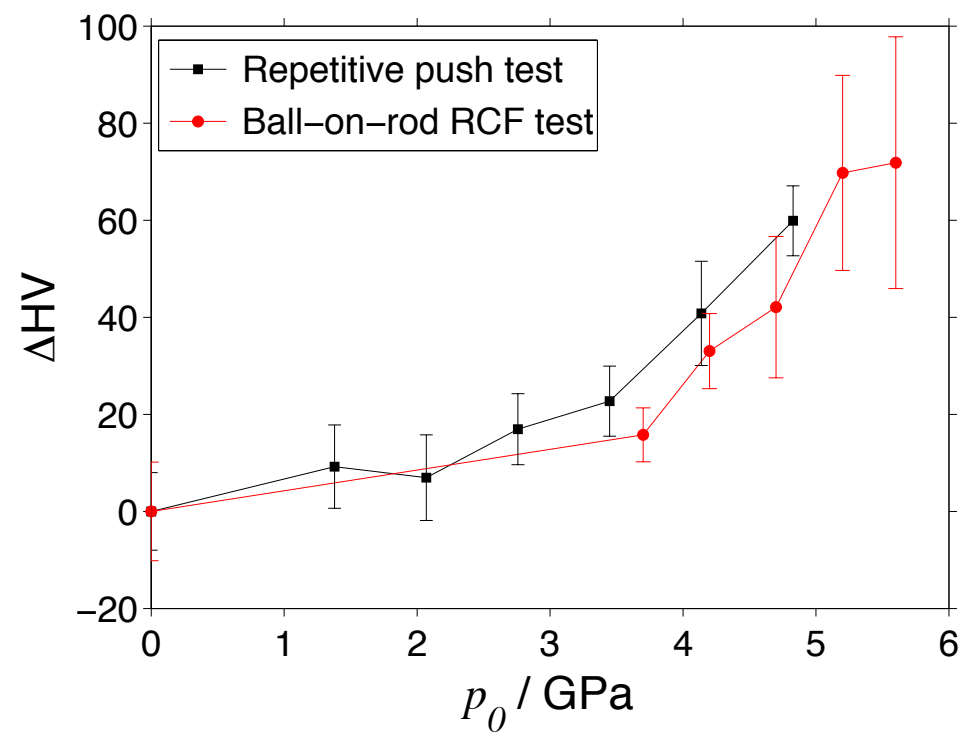

Figure 17: Hardness increase acquired during the repetitive push tests after $N=5 \times 10^{5}$ cycles, and the ball-on-rod rolling contact fatigue tests after $N=10^{8}$ cycles [18]. 
Table 1: Composition of the 100Cr6 steel used in the study (all in wt\%). Fe is balanced accordingly.

\begin{tabular}{cccccccc}
\hline $\mathrm{C}$ & $\mathrm{Cr}$ & $\mathrm{Mn}$ & $\mathrm{Si}$ & $\mathrm{Cu}$ & $\mathrm{Ni}$ & $\mathrm{Mo}$ & $\mathrm{Al}$ \\
\hline 0.97 & 1.38 & 0.28 & 0.28 & 0.21 & 0.18 & 0.06 & 0.04 \\
\hline
\end{tabular}

Table 2: Corresponding maximum resolved shear stress $\left(\tau_{r s s, \text { max }}\right)$ and contact pressure $\left(p_{0}\right)$ for the maximum pressure $\left(p_{\max }\right)$ used in the repetitive push test.

\begin{tabular}{ccc}
$p_{\text {max }} / \mathrm{GPa}$ & $\tau_{\text {rss }, \text { max }} / \mathrm{GPa}$ & $p_{0} / \mathrm{GPa}$ \\
\hline 1.0 & 0.34 & 1.38 \\
1.5 & 0.52 & 2.07 \\
2.0 & 0.69 & 2.76 \\
2.5 & 0.86 & 3.45 \\
3.0 & 1.03 & 4.14 \\
3.5 & 1.21 & 4.82 \\
\hline
\end{tabular}

Table 3: Fitting parametres in Eqs. 4 and 5 for different conditions.

\begin{tabular}{cccc}
\hline$p_{\max }$ & $b_{1}$ & $b_{2}$ & $b_{3}$ \\
\hline 1.0 & $3.625 \times 10^{-4}$ & $4.978 \times 10^{-1}$ & $3.225 \times 10^{-4}$ \\
1.5 & $8.244 \times 10^{-4}$ & $1.934 \times 10^{-1}$ & $4.308 \times 10^{-4}$ \\
2.0 & $1.393 \times 10^{-3}$ & $1.961 \times 10^{-1}$ & $5.431 \times 10^{-4}$ \\
2.5 & $1.177 \times 10^{-3}$ & $4.010 \times 10^{-1}$ & $5.258 \times 10^{-4}$ \\
3.0 & $1.114 \times 10^{-3}$ & $5.063 \times 10^{-1}$ & $5.315 \times 10^{-4}$ \\
3.5 & $1.381 \times 10^{-3}$ & $4.283 \times 10^{-1}$ & $6.294 \times 10^{-4}$ \\
\hline
\end{tabular}

